\title{
Experimental Ultrasonic Assessment of Steel Induction Hardening by Measuring Two Distinct Times-of-Flight
}

\author{
W.Chen, A.N.Kalashnikov*, R.E.Challis, M.G.Somekh \\ Department of Electrical and Electronic Engineering, Nottingham University, Nottingham, NG9 7EB, United Kingdom \\ *Corresponding Author: alexander.kalashnikov@nottingham.ac.uk
}

Copyright (C) 2013 Horizon Research Publishing All rights reserved.

\begin{abstract}
Ultrasonic NDE of case hardening of steel parts presents an attractive alternative to destructive assessment still used in industry. This experimental study showed that the ultrasonic assessment could be conducted by measuring two average times of flight across different dimensions of a hardened test sample. The consistently observed difference in the corresponding average ultrasound velocities was above the estimated accuracy of the measurements, and the scattered individual calculated values formed distinct clusters. The results were obtained by using a high resolution data acquisition instrument with the equivalent sampling frequency of $1.5 \mathrm{GHz}$ and 512 averages, and differential measurements of the time of flight by determining zero crossings of the echo waveforms.
\end{abstract}

Keywords Case Hardening, Ultrasonic Non-Destructive Evaluation, Time-of-Flight

\section{Introduction: Motivation for Development of Ultrasonic NDE for the Assessment of Steel Surface Hardness}

Steelmaking became a milestone in the history of human civilization. Ferrous alloys are still most important engineering materials used everywhere. The properties of these alloys depend on their chemical composition (steels are differentiated by as little as $0.1 \%$ by weight of the ingredients, most notably carbon), macrostructure (size of individual iron crystals - grains) and microstructure (body-centered cubic BCC or face-centered cubic - FCC) [1-3].

Use of ferrous alloys of higher quality is restricted by their costs. Additionally, some desirable material qualities are contradictory. Most notably high hardness of a ferrous alloy material (resistance to indent and wear) associates with low ductility (ability to plastically strain without fracture) usually. Therefore an application specific compromise is to be reached when selecting or manufacturing steel parts to avoid excessive brittleness. Required composition is prepared by dissolving alloying ingredients at particular temperatures according to the relevant (e.g., iron-carbon) phase diagram [1-3].

Another approach widely used in industry is to combine hardness and ductility by hardening the part's surface only and leaving the core ductile. This surface hardening can be achieved by diffusion into the surface layer atoms caused an increase in hardness (e.g., carburizing, nitriding etc) [1-3].

Alternatively a thermal treatment can be applied to change the surface layer's microstructure by rapidly heating it to the austenite state (FCC) then rapidly cooling it to the martensite (BCC). Rapid heating is required in order not to affect the ductile core. This heating can be achieved by placing a metal part into a strong electric field that does not penetrate deep into metals. The induced surface electrical currents heat the surface only. Rapid cooling (quenching) to room temperatures is necessary for the transformation of austenite into martensite instead of pearlite or bainite. This need can be seen from the time-temperature-transformation (TTT) curve. The required cooling can be achieved by placing the part into an oil or water bath quickly [1-3].

The described above induction case hardening is suitable for rapid mass manufacturing of parts with complex geometries. That is why it is used widely, e.g., in automotive industry for manufacturing of car engine crankshafts. The strength and frequency of the electric field in the induction furnace along with the exposure time can be controlled to achieve the desired hardened layer depth and surface hardness [2].

The quality control of the manufactured parts involves measurement of the obtained surface hardness. This can be done by applying a set force to an indenter of a particular shape, and measure the resulting indentation area (HB Brinell, HV Vickers, HK Knoop hardness tests) or depth (HRA, HRB, HRC Rockwell hardness tests). The Brinell hardness is proportional to the ultimate tensile strength of the material under test whilst the other tests use specific scales $[4]$.

All these tests are time consuming and leave marks on the surface. Because of this the quality control is carried out by taking a set percentage of samples out of the production line and testing them destructively by cutting to halves first. A 
major car manufacturer that cannot be named because of the confidentiality agreement inquired about the possibility of using ultrasonic NDE for quality control of the car engine crankshafts. The initial research focused on the possibility of using surface waves to determine the depth of the hardened layer, but the resolution of the instrumentation used did not allow development of a convincing quality control procedure [5]. The problem was revisited recently using higher resolution instrumentation developed in our laboratory [6] with more success.

The paper is organized as follows. Section 2 discusses different approaches to ultrasonic NDE of surface hardness and results obtained. Section 3 introduces an approach that allows quality assessment based on two separate time-of-flight measurements. Section 4 describes the experimental setup and samples. Section 5 presents the experimental waveforms and results of their processing. Section 6 contains discussion of the issues raised in the paper that is concluded by Section 7 .

\section{Relating Ultrasonic Velocity to Surface Hardness: Some Previously Developed Techniques and Results}

Although some destructive tests show a clear interface between the hardened case and the core, the depths of the hardened case seems difficult to assess non-destructively. This motivated research of several suitable ultrasonic NDE techniques.

The case-core interface sometimes can be located by using the conventional pulse-echo technique (fig.1a). The estimate of the time of flight between the excitation and the echo from the interface is converted into the case depth if the longitudinal velocity of ultrasound in the hardened layer is known [7]. This technique is applicable for case depths above $10 \mathrm{~mm}$ [8] that is too deep for crankshafts.

Another technique applies angular incidence of longitudinal waves which are converted into transverse waves at the boundary of the test piece (fig. $1 \mathrm{~b}$ ). The time delay of the edge of the backscatter signal is used for the case depth estimation then [9]. This technique requires proper calibration of the corresponding instruments, some of the latter are available commercially. It is usable in the range from 2-4 $\mathrm{mm}$ up to $15 \mathrm{~mm}$ [8].

Incident longitudinal ultrasonic waves can be efficiently converted into surface waves (predominantly Rayleigh waves for samples much thicker than the wavelength) at some specific angles of incidence. The test piece is placed into a liquid tank, and is subjected to an ultrasonic beam at different angles of incidence (and sometimes at different frequencies), fig.1c $[8,10,11]$. The angles resulted in strong signals from the pick up receiver are related to the case depth. The disadvantages of the method are the necessity of submerging the test piece into a liquid and indirect relationship between the critical angle(s) and the case depth.
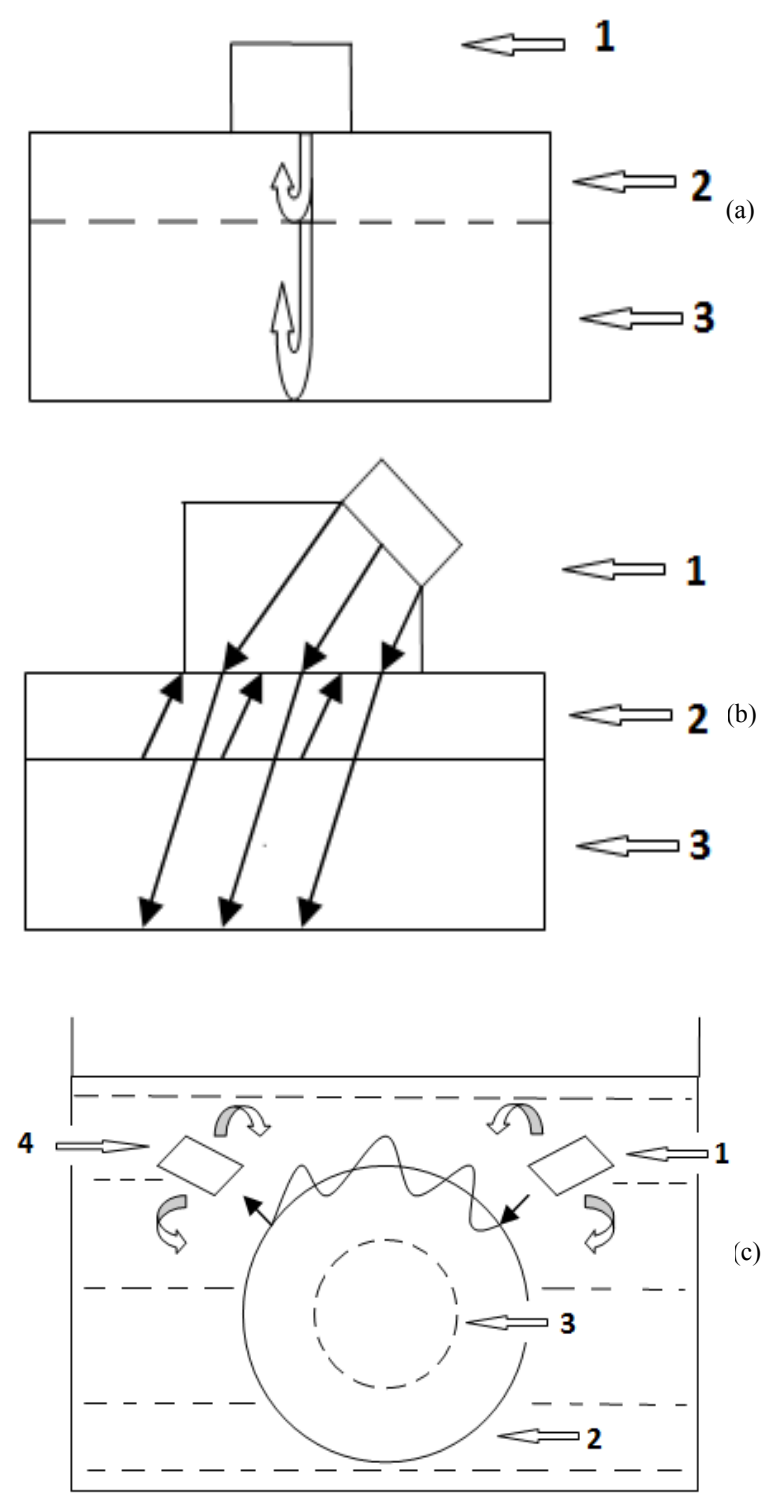

Figure 1. Use of conventional ultrasonic transducers for assessing hardened layer: $\mathrm{a}$ - pulse echo test; $\mathrm{b}$ - backscatter test; $\mathrm{c}$ - critical angle test ( 1 - the ultrasonic transducer, 2 - the hardened layer, 3 - the core)

Recent developments of these methods utilise laser ultrasound to excite either surface (fig.2a, [12,13]) or longitudinal (fig 2.b, $[12,14]$ ) waves remotely that makes in process monitoring possible.

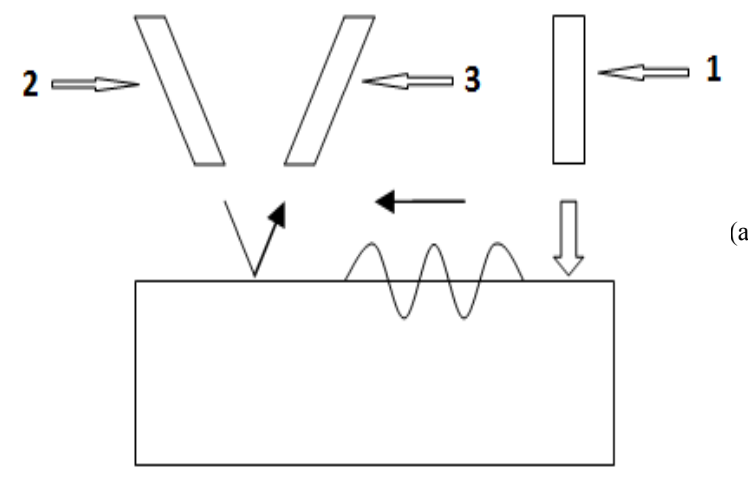

(a) 


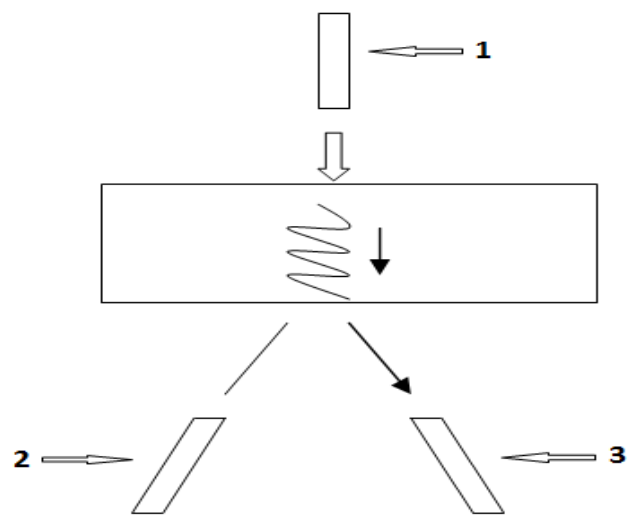

(b)

Figure 2. Use of laser ultrasound for assessing hardened layer using (a) surface waves and (b) bulk waves ( 1 - laser pulser, 2 - pick up laser, 3 photo detector for knife edge detection)

For the post production NDE the preparation of the test samples is very important in order to establish robust NDE technique that allows detecting the right condition of a particular test piece in spite of inevitable statistical variations among the test pieces. The same test piece can possess different surface hardness if its parts were quenched at different cooling rates $[11,15]$ or grinded at some angle to the surface $[9,16]$. Such a test piece can be used to determine the relationship between the surface hardness and ultrasound velocity for this test piece considered as a representative one. A set of test pieces can be taken straight from a production line to find the aggregate trend and the boundaries for production variations statistically [17-19]. Finally, several test pieces can be prepared in order to vary a particular property only whilst keeping the other properties the same (e.g., varying test piece structure $[14,20]$ or carbon content [21] or process parameters $[14,22])$.

The results for experimentally determined ultrasound velocities for test pieces with different surface hardness are presented in table 1 . It shows that the ultrasound velocity varies at around $1 \%$ only for even substantial changes of the surface hardness. Both positive and negative correlations of the two were observed experimentally. The possible opposite signs of the correlation coefficients would suggest an existence of cases when the ultrasonic velocity is independent on the surface hardness, and these were observed experimentally for some steels [8].

Table 1. Experimental results for simultaneous measurements of the surface hardness and ultrasonic velocity in various steels

\begin{tabular}{|c|c|c|c|c|}
\hline Steel type & $\begin{array}{c}\text { Wave type, } \\
\text { velocity range } \\
(\mathrm{km} / \mathrm{s}) \text { and variation }\end{array}$ & Hardness range & Relation & Ref \\
\hline \multicolumn{5}{|c|}{ Hardened steels } \\
\hline $\begin{array}{c}1060 \text { one side } \\
\text { induction hardened }\end{array}$ & Not stated & 50-20 HRC & $\mathrm{N}$ & 9 \\
\hline 50B46 quench hardened & R 2.95..3.00 1.7\% & $650.300 \mathrm{HK}$ & $\mathrm{N}$ & 11 \\
\hline 1043 induction hardened & R 2.928..2.974 1.5\% & $50 . .25 \mathrm{HRC}$ & $\mathrm{N}$ & 21 \\
\hline Not stated & $\begin{array}{l}\mathrm{L}(5.915 \text { hardened, } \\
5.835 \text { plain }) 1.3 \%\end{array}$ & Not stated & $\mathrm{N}$ & 8 \\
\hline $\mathrm{XC} 38 \mathrm{H} 2$ quench hardened & L $6.2 . .6 .11 .7 \%$ & $600 . .200 \mathrm{HV}$ & $\mathrm{P}$ & 15 \\
\hline \multicolumn{5}{|c|}{ Plain steels } \\
\hline Plain carbon hot rolled sheets $09 \mathrm{G} 2 \mathrm{~S}$, many samples & R $2.872 . .2 .8860 .3 \%$ & $1880<\mathrm{HV}<2160 \mathrm{MPa}$ & $\mathrm{P}$ & 19 \\
\hline $81 \mathrm{xx}$ carbon $0.25 . .1 .03 \%$ & L $5.90 . .5 .860 .7 \%$ & 39..60 HRC & $\mathrm{N}$ & 21 \\
\hline Rollers for reinforced concrete, 23 samples & L 5.240..5.200 1.2\% & 64..56 HRC & $\mathrm{P}$ & 17 \\
\hline Various steels, 173 samples & L 5.240..5.140 1.9\% & $300 . .700 \mathrm{HV}$ & $\mathrm{N}$ & 18 \\
\hline \multicolumn{5}{|c|}{ Modified or coated steels } \\
\hline $\begin{array}{l}\text { Ti-modified austenitic stainless steel, different } \\
\text { annealing }\end{array}$ & L 5.9..5.7 & $140 . .265 \mathrm{HV}$ & $P$ & 22 \\
\hline Chromium coated steel & R 3.0..3.7 & $550 . .800(?)$ & $P$ & 13 \\
\hline
\end{tabular}

\section{NOTES:}

$\mathrm{L}$ - longitudinal $\quad \mathrm{R}$ - Rayleigh

$\mathrm{P}$ - positive correlation $\mathrm{N}$ - negative correlation 


\section{Assessment of Case Hardening by Measuring Two Distinct Times-of-Flight}

Let us consider a rectangular bar which surface was hardened uniformly. Neglecting the corner effects the cross section of the bar can be represented as shown in fig. 3 with the hardened layer of width $x$ and average longitudinal ultrasound velocity of $c_{h}$ and the unhardened core with the average longitudinal ultrasound velocity of $c_{0}$.

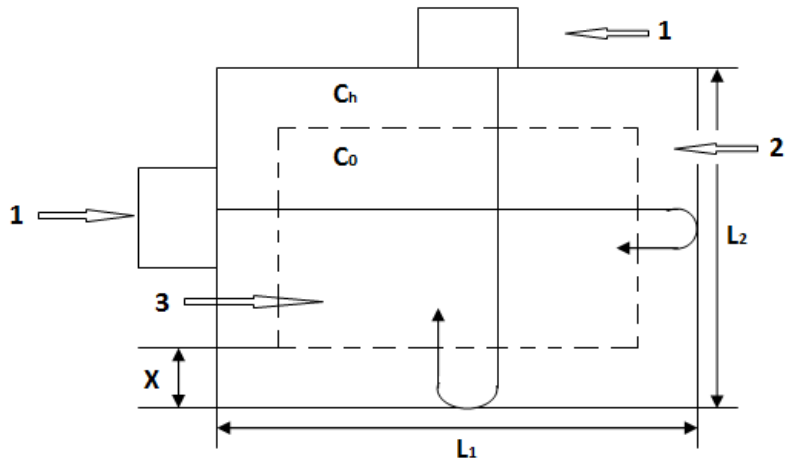

Figure 3. Measurement arrangements $(1-$ ultrasonic transducers, 2 hardened layer, 3 - core)

The time of flight of ultrasound in the pulse-echo mode for two transducers shown equals

$$
\left\{\begin{array}{l}
t_{1}=\frac{l_{1}-2 x}{c_{0}}+\frac{2 x}{c_{h}} \\
t_{2}=\frac{l_{2}-2 x}{c_{0}}+\frac{2 x}{c_{h}}
\end{array} .\right.
$$

Taking the difference of the above equations and re-arranging yields

$$
c_{0}=\frac{l_{1}-l_{2}}{t_{1}-t_{2}}
$$

or, when $N$ measurements were made across the different dimensions of the sample,

$$
c_{0}=\frac{\sum_{i=1}^{N} l_{1 i}-\sum_{i=1}^{N} l_{2 i}}{\sum_{i=1}^{N} t_{1 i}-\sum_{i=1}^{N} t_{2 i}} .
$$

Taking the sum of the parts of the eqn.1 and re-arranging yields

$$
x\left(\frac{1}{c_{0}}-\frac{1}{c_{h}}\right)=1 / 4\left[\frac{\left(l_{1}+l_{2}\right)}{c_{0}}-\left(t_{1}+t_{2}\right)\right],
$$

or, when $\mathrm{N}$ measurements are considered,

$$
x\left(\frac{1}{c_{0}}-\frac{1}{c_{h}}\right)=1 / 4 N\left[\frac{\left(\sum_{i=1}^{N} l_{1}+\sum_{i=1}^{N} l_{2}\right)}{c_{0}}-\left(\sum_{i=1}^{N} t_{1}+\sum_{i=1}^{N} t_{2}\right)\right] .
$$

The right parts of eq. (3), (5) can be calculated out of mechanical (for lengths) and ultrasonic (for timings) measurements, but the left part of the eq. (5) depends on two unknown variables and is measured in time units. Let us estimate its numerical value.

The case hardening process aims to achieve the case depth of above $1 \mathrm{~mm}$, and the longitudinal ultrasound velocity in steels can be taken as $5,000 \mathrm{~m} / \mathrm{s}$ for a crude estimate. As table 1 shows the longitudinal ultrasound velocity in the hardened layer differs from that in the core in an order of $1 \%$. Using these values one can estimate the numerical value of the left part of the eq. $(4,5)$ as $4 \mathrm{~ns}$. This value amounts to a short time but it can be measured by modern instruments, especially taking into account the possibility of reducing measurement uncertainties by taking several measurements, eq. (5). The left part of the eq. $(4,5)$ increases when the thickness of the hardened layer increases, and when the ultrasonic velocity in the hardened layer differs more from this velocity in the core. Consequently the time value calculated from the experimental measurements using the right part of the eq. (5) seems to represent an estimate of changes of the test piece due to its case hardening, and could allow development of the "go" and "no-go" gauges for the quick post-production quality control of case hardening.

\section{Test Samples, Experimental Procedures and Instrumentation Used}

The test samples were prepared from five metal bars from the same material with notionally the same dimensions that underwent the same induction hardening. Using the same factors affecting the surface hardness allowed assessing possible manufacturing variations. Additionally, the top surface of four hardened samples received from the manufacturer was grinded to a different depth in order to achieve some variations of the surface hardness (as it should decrease towards the core) and presumably ultrasound velocity (fig.4).

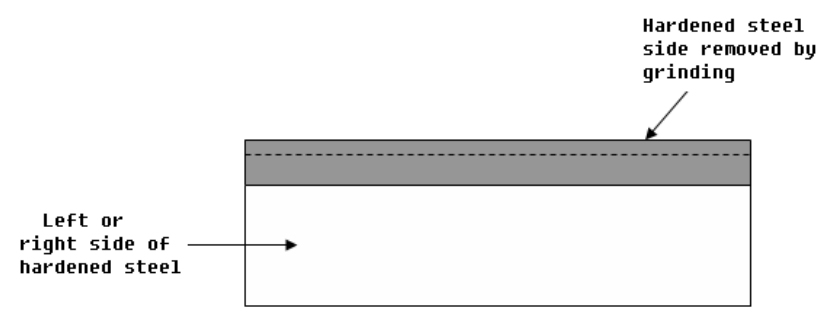

Figure 4. Hardened layer, core and part of the hardened layer removed by grinding

The average dimensions of the prepared samples are given in the table 2. These dimensions were measured by a digital Vernier calliper, a micrometer with the range $50-75 \mathrm{~mm}$ and a micrometer with the range $0-25 \mathrm{~mm}$ respectively. The resolution of all the instruments was $10 \mu \mathrm{m}$. The temperature in the laboratory during the measurements was within $19 . .21^{\circ} \mathrm{C}$ which allows neglecting the heat expansion. 
Table 2. Average dimensions of the test samples after grinding

\begin{tabular}{|c|c|c|c|c|c|c|}
\hline Sample No & 1 & 2 & 3 & 4 & 5 \\
\hline Dimensions (mm) & $\begin{array}{c}200 \times 50.68 \times \\
17.60\end{array}$ & $\begin{array}{c}200 \times 50.71 \times \\
17.92\end{array}$ & $\begin{array}{c}200 \times 50.75 \times \\
18.30\end{array}$ & $\begin{array}{c}200 \times 50.71 \times \\
18.53\end{array}$ & $\begin{array}{c}200 \times \\
50.73 \times \\
18.99\end{array}$ \\
\hline $\begin{array}{c}\text { Grinding } \\
(\mathrm{mm})\end{array}$ & -1.39 & -1.07 & -0.69 & $-0.46 \quad$ No grinding \\
\hline
\end{tabular}

Two ultrasonic records were taken at each test point (except for the last area where only one record was taken due to peculiarities of the back end software).

Five test areas were marked on the top and the side of samples 1-4, 6 and four areas on the top and side of sample 5 due to its lower length. The widths of the samples in the marked areas were measured three times, and the averages of these three measurements were used for later ultrasound velocity calculations.

The ultrasound velocity was measured in the pulse echo mode by placing a $20 \mathrm{MHz}$ ultrasound transducer (Panametrics, Videoscan V316) in the marked areas one after one in the direction shown by an arrow (fig.5,6).The measurements were repeated three times for the top surface, and two times for the side surface of every sample.

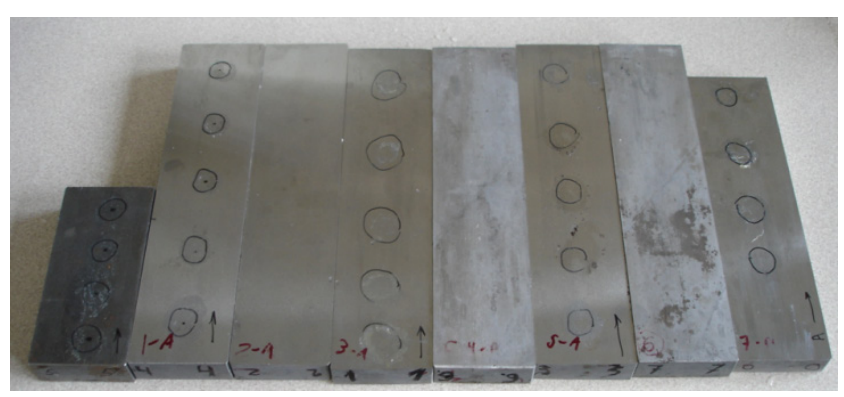

Figure 5. Photograph of the samples used

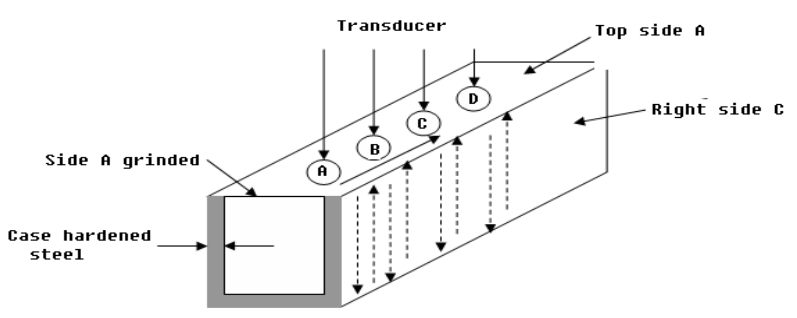

Figure 6. Taking ultrasonic echo records from the grinded top side

The ultrasonic echoes were recorded using the FPGA based instrument that combines waveform averaging and accurate interleaved sampling [6]. The instrument was implemented using a Xilinx Xtreme DSP board housed in a small form factor PC. NDT Solutions pulser receiver was used as the analogue front end. The excitation voltage was set to $100 \mathrm{~V}$, and the receiver gain was adjusted to utilise the whole range of the on board ADC. The waveforms were sampled by the $\mathrm{ADC}$ at $100 \mathrm{MHz}$ with the interleaving factor of 15. This setting provided the equivalent sampling frequency of $1.5 \mathrm{GHz}$. The number of averages used was 512 that increased the signal-to-noise ratio by about $27 \mathrm{~dB}$. The ultrasonic transducer was used in a pulse-echo mode without a shoe, and was pressed against the test point by hand.

\section{Recorded Waveforms and Processed Results}

Typical waveforms recorded for all the test points on the top surface are presented in fig.7. It shows the first and subsequent echoes only. The time-of-flight was determined by the time delay between the first and the second echoes without considering the excitation pulse. This allowed cancelling any additional delays related to the propagation of the ultrasonic pulses in the transducer-sample couplant etc. The recorded waveforms did show variability in their amplitudes, time positions and shape for the first and the second echoes (fig.8ab). Differences in timings and amplitudes were observed mainly due to differences in the surface roughness and hand pressure at different test points. The latter differences also affected the shape of the first echo. The shapes of the second echoes, especially tails, were more regular than these of the first echoes because of higher attenuation of ultrasonic signals at high frequencies in steels. This is equivalent to low pass filtering of ultrasonic signals during their propagation.

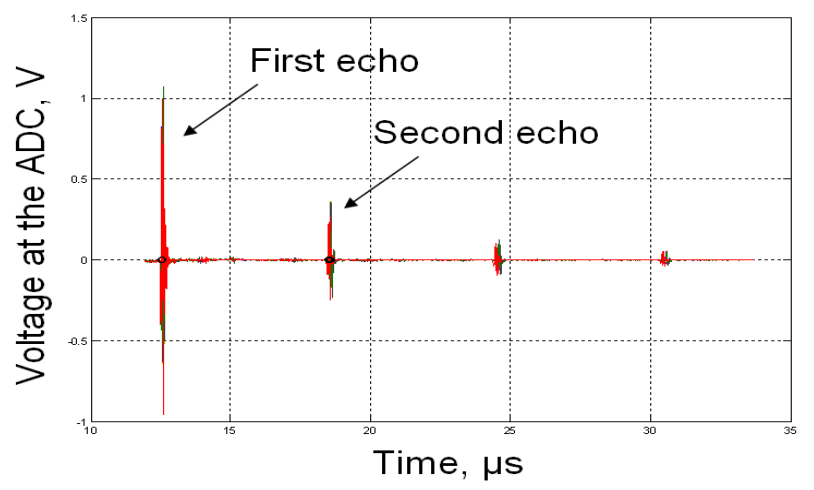

Figure 7. An example of recorded ultrasonic echoes on top surface

Examples of the experimental waveforms for the side surface are presented in fig.9. The main differences compared to fig. 7,8 are the longer delay between the first and second echo, clearer presence of parasitic signals, alterations in shape due to longer propagation distance thus extra filtering and less scattered positions of the first echo signals possibly because of flatter surface at the side. 


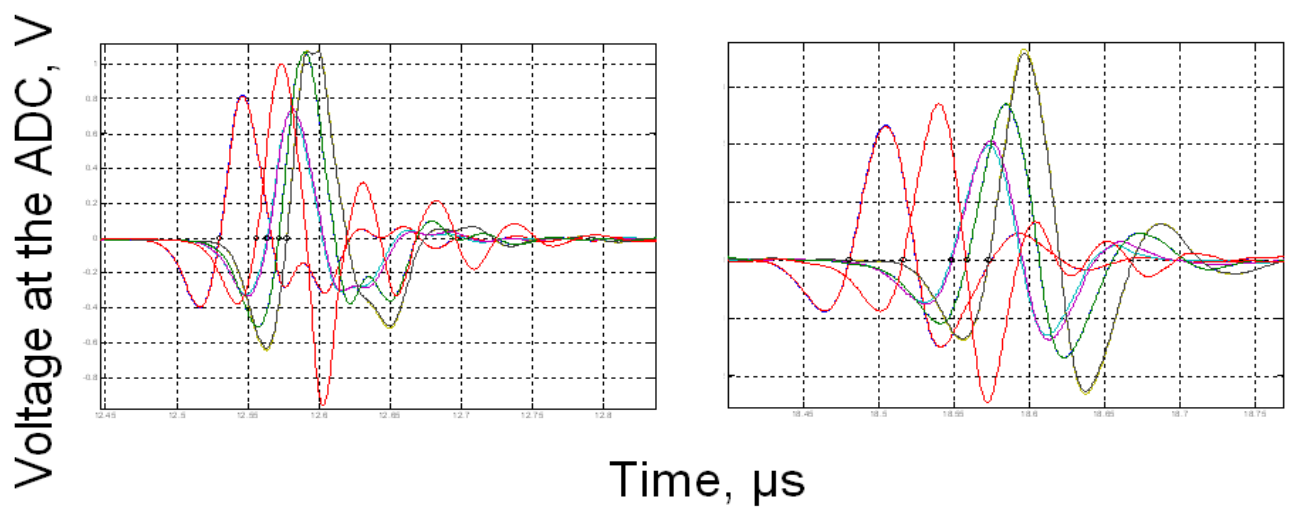

Figure 8. Magnified from fig.7 first (left) and second (right) echo pulses at the top surface

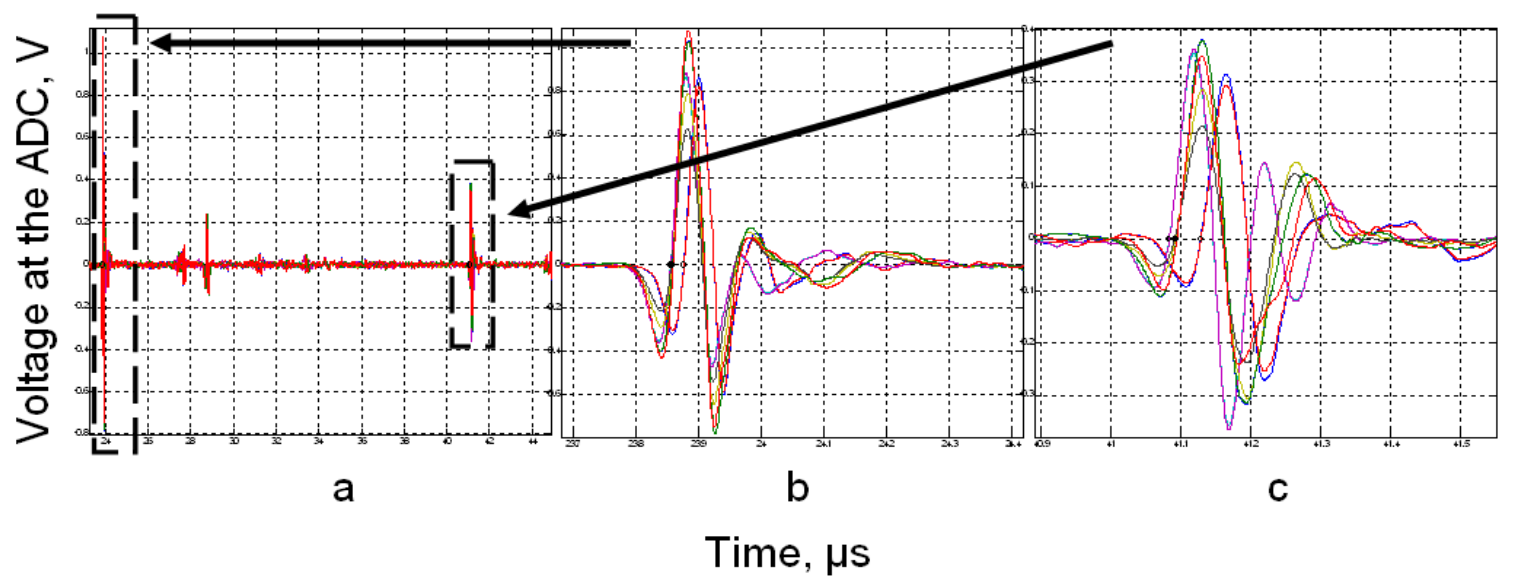

Figure 9. Example waveforms for the side surface: overall picture showing some parasitic signals between the first and second echoes (a), first echo (b) and second echo (c)

The time of flight was determined using zero crossings of the first and second echoes. First transition between the negative and positive lobes of the echoes was used for both. Each echo position was calculated using linear interpolation between the two adjacent samples with different signs (fig.10), and the delay was calculated by subtraction.

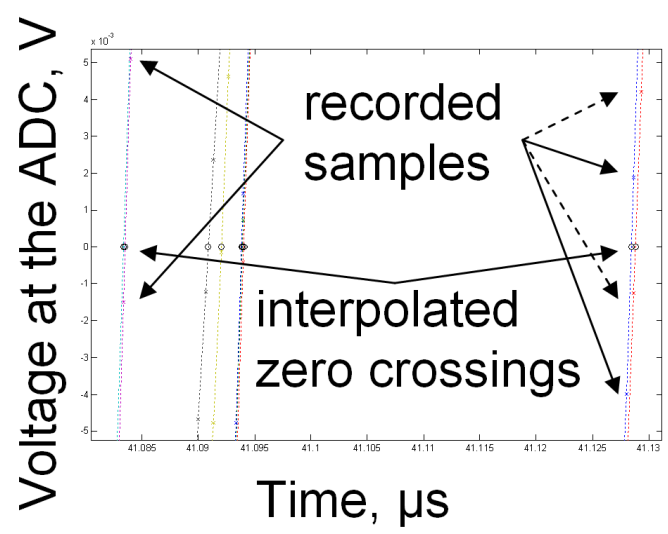

Figure 10. Time position of echoes found by using linear interpolation (crosses - waveform samples, lines - linear interpolation between the latter, circles - sought after zero crossings)

This ensured an increase in time resolution compared to the equivalent sampling frequency that can be estimated at 3 to 10 times from past experience.

The average width at the each test point doubled (for pulse echo mode) was divided by the time of flight determined as above for every experimental record taken. The results obtained are presented in fig. 11 by points. In fig. 11 the hardened samples are ordered by the increase in the depth of grinding from left to right rather than by their numbers in table 2.

Fig.11 shows a clear separation between the calculated ultrasound velocities for the top (inside the dashed rectangle) and side (outside the rectangle) surfaces despite the notable scatter. The legend over fig. 11 explains the order at which the all the calculated values were put on the graph.

Table 3 presents processed aggregates for the calculated velocity data set - mean ultrasound velocity and its variability quantified as the ratio of standard deviation (STD) to the mean value.

An immediate outcome from the dataset is that ultrasound velocity in the hardened surface layer increased. This is because the velocity calculated for the top surface (relatively thicker hardened layer) was higher than that for the side surface (relatively thinner hardened layer). The numerical estimate for the ultrasound velocity for the core was calculated using (3), and found equal to $5.8722 \mathrm{~m} / \mathrm{s}$. That was somehow lower than the velocity estimated for the 
control sample 6 of untreated mild steel where the velocity was estimated at $5.9017 \mathrm{~km} / \mathrm{s}(\mathrm{STD} /$ mean $=0.02 \%)$. The manufacturer who gave the mild steel sample 6 was unwilling to confirm neither the same sort of steel for all the samples nor the steel sort difference between the mild steel and the hardened samples.

These experimental results do not contradict results published previously, and do not dismiss the possibility for ultrasonic NDE of case hardening by measuring two distinct times-of-flight.

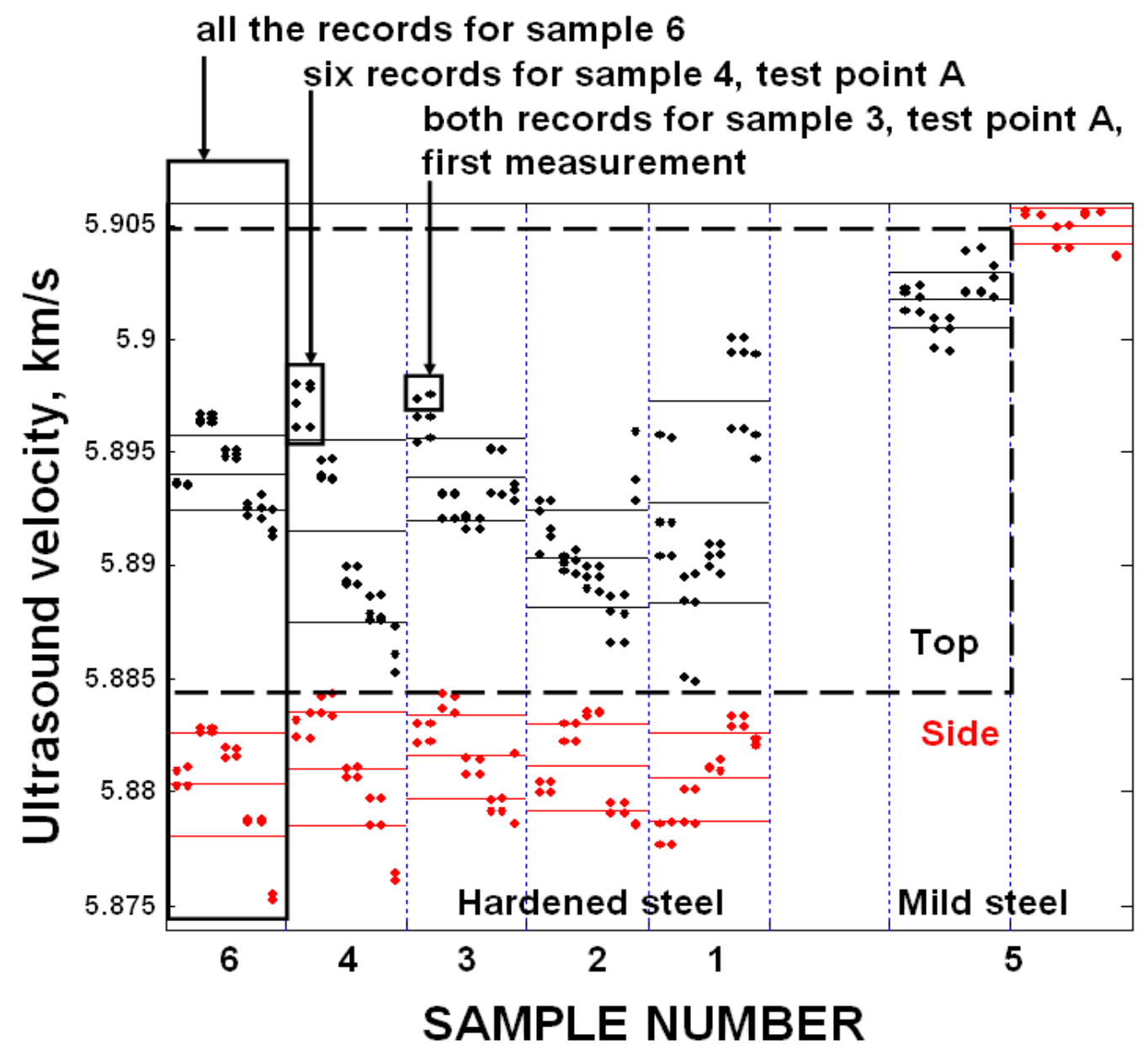

Figure 11. Ultrasound velocities calculated from the experimental data (the horizontal lines show mean value plus STD, mean value, mean value minus STD from top to bottom respectively)

Table 3. Aggregate results for the calculated ultrasound velocity

\begin{tabular}{|c|c|c|c|c|c|c|}
\hline \multirow{2}{*}{$\begin{array}{c}\text { Sample } \\
\text { number }\end{array}$} & \multicolumn{3}{|c|}{ Mean velocity, km/s } & \multicolumn{3}{c|}{ STD / mean, \% } \\
\cline { 2 - 7 } & Top & Side & Ratio & Top & Side & Ratio \\
\hline 6 & 5.8941 & 5.8804 & 1.00233 & 0.028 & 0.039 & 0.717949 \\
\hline 4 & 5.8915 & 5.8811 & 1.001768 & 0.069 & 0.043 & 1.604651 \\
\hline 3 & 5.8939 & 5.8816 & 1.002091 & 0.031 & 0.031 & 1 \\
\hline 2 & 5.8903 & 5.8811 & 1.001564 & 0.037 & 0.033 & 1.121212 \\
\hline 1 & 5.8928 & 5.8807 & 1.002058 & 0.075 & 0.033 & 2.272727 \\
\hline $\begin{array}{c}\text { Column } \\
\text { averages }\end{array}$ & $\mathbf{5 . 8 9 2 5 2}$ & $\mathbf{5 . 8 8 0 9 8}$ & $\mathbf{1 . 0 0 1 9 6 2}$ & $\mathbf{0 . 0 4 8}$ & $\mathbf{0 . 0 3 5 8}$ & $\mathbf{1 . 3 4 0 7 8 2}$ \\
\hline
\end{tabular}




\section{Accuracy and Variation of the Velocity Estimates}

The accuracy of velocity determination was estimated using the resolution of length and time of flight measurements. The former was equal to the resolution of the micrometers that was $10 \mu \mathrm{m}$. At the worst case the length measured was $20 \mathrm{~mm}$ thus the relative accuracy was

$$
\varepsilon_{l}=\frac{10 \mu m}{20 m m} \times 100 \%=0.05 \% .
$$

This value was reduced further by taking three measurements. The related accuracy of the time measurements can be estimated by dividing the time resolution to the overall time of flight:

$$
\varepsilon_{t}=\frac{1 / 1.5 G H z}{\frac{20 m m \times 2}{6000 m / s}} \times 100 \% \approx 0.012 \%,
$$

where multiplier 2 relates to pulse-echo mode, the ultrasound velocity was taken as $6000 \mathrm{~m} / \mathrm{s}$, and the time resolution was equated to the reciprocal of the equivalent sampling frequency. This value was reduced further by taken 6 records for most test points, and using linear interpolation to determine the time crossing. Consequently the relative accuracy of the obtained experimental velocity was about $0.05 \%+0.01 \%=0.06 \%$ or $3.6 \mathrm{~m} / \mathrm{s}$. If the ultrasound velocity in the hardened layer changes by $1 \%$ comparing to the core, and the length of this layer(s) is about $10 \%$ of the ultrasonic pathway, the relative change in the ultrasound velocity measured for this sample will be

$$
\varepsilon_{v}=1 \% \times 10 \%=0.1 \%,
$$

which corresponds to around $5 \mathrm{~m} / \mathrm{s}$ of the ultrasound velocity. Therefore the instrumentation used could be considered accurate enough to detect the change of the ultrasound velocity caused by forming the hardened layer if applicable.

The dimensions of the samples were measured by two separate micrometers that could have been biased differently. The calculations of the ultrasound velocity for the control sample 5 shows that notionally the same ultrasound velocities across the top and side surfaces were different by $3.3 \mathrm{~m} / \mathrm{s}$ on average. (This value agrees with the accuracy of calculated ultrasound velocity estimated above.) However fig. 11 shows that higher velocity was estimated for the side surface that was opposite to what was observed for the hardened samples. Consequently if a permanent bias existed between the two micrometers (and/or signal processing procedures) used, it would lead to reducing the observed difference between the average ultrasound velocities for the considering surfaces. Despite this possibility the clusters of the velocity estimates for the side and top surfaces were clearly separated (fig.10).

The standard deviation of the estimates of the ultrasound velocity was found considerably bigger for the top side on average $(0.048 \%$ versus $0.036 \%$, table 3$)$. However fig. 10 and table 3 show that for the samples $6,3,2$ the scatter of estimated velocities for both surfaces looked similar. If these standard deviations were solely dependent on the resolutions of the instruments, the side measurements across $50 \mathrm{~mm}$ length would produce STDs smaller by the factor of $\sqrt{50 \mathrm{~mm} / 20 \mathrm{~mm}} \approx 1.6$ that significantly exceeds the calculated value of 1.34 (table 3 ). Moreover, the scatter of the estimated velocities should have been consistent across all the samples which is absent from fig. 10 for the top side. On this basis it is reasonable to assume that the STD of experimentally estimated ultrasound velocities depends on the length of the ultrasonic pathway. The higher STDs calculated for the top surface seem to be related to imperfectness of the grinded surface and local heating of the samples in the grinding process [2, ch.26.1.2].

\section{Conclusions}

Ultrasonic NDE of hardened steel parts can be conducted by measuring ultrasound velocity of the bulk wave propagated through both the hardened layer(s) and the core, or the surface wave. According to the literature, these velocities can both increase and decrease along with the increase in hardness for different steels. Therefore for some steels the dependence might be very small or even absent thus difficult if possible to detect. The dependence of the surface hardness on the ultrasonic velocity needs to be studied for different sorts of steel separately, using sets of test samples hardened differently rather than cold working a single sample.

The change of ultrasound velocity in the hardened layer seems to be around $1 \%$ if applicable, and around $0.1 \%$ for the average bulk ultrasound velocity across realistic hardened samples. Detection of these changes requires use of high resolution instrumentation, substantial dimensions of the samples and repetitive measurements with further statistical processing.

The estimation of the time of flight of ultrasound in hardened steel samples is complicated by the difficulty of maintaining the same acoustic contact between the probe and the sample across all the test points, and by changes of the shape of the propagating waveform along with the distance travelled. The former factor was eliminated by estimating the delay between the first and second echo pulses (rather than the excitation and first echo pulses). The latter factor was mitigated by considering first zero crossings of the echoes rather than their cross-correlation or centre-of-gravity estimates.

The present study used ultrasonic assessment of steel induction hardening by measuring two distinct times-of-flight. This assessment was applied to rectangular bars that were grinded to different depth after the treatment. Measurements across the ultrasonic pathways of $40 \mathrm{~mm}$ and $100 \mathrm{~mm}$ (the sample dimensions were doubled here because of the pulse echo mode used) showed a clear difference in the average ultrasound velocities. This difference might be used 
to develop a "go"-"no go" gauge for NDE of the induction hardened parts after production.

The reported experiments employed rectangular samples whilst many hardened parts possess curved surfaces (like crankshafts). Curved parts may not be necessarily suitable for the described technique. However it seems viable to place a rectangular steel bar made from the same material as the hardened part close to the latter and subject both to the same induction hardening treatment. The bar can be inspected afterwards as described above to gauge the result of the treatment, and destructively tested if necessary. Although this modification requires the use of additional rectangular samples to be scrapped after the test and it is not applied to the hardened samples directly, it nevertheless seems advantageous to the present practices. At present a small percentage of crankshafts is tested destructively whilst the described procedure allows at least an increase of this percentage (if $100 \%$ testing is not viable for time/cost reasons) and further use of the parts checked because they are not damaged by testing.

\section{REFERENCES}

[1] E.P.DeGarmo, J.T.Black and R.A.Kohser, Materials and processes in manufacturing, $8^{\text {th }}$ ed., John Wiley and sons, 1999.

[2] M.P.Groover, Fundamentals of modern manufacturing: materials, processes and systems, John Wiley and sons, 1999.

[3] W.D.Callister Jr., Materials science and engineering: an introduction, $5^{\text {th }}$ ed., John Wiley and sons, 2000.

[4] Methods of measuring hardness and equivalent hardness conversion tables, available online http://tinyurl.com/kk59ar, accessed Sept 2013.

[5] G.A.Sosa Lopes, Ultrasonic non-destructive evaluation of the surface layer of case hardened steel, MRes thesis, Nottingham University, 2005.

[6] A.N.Kalashnikov, V.G.Ivchenko, R.E.Challis and B.R.Hayes-Gill, "High-accuracy data acquisition architectures for ultrasonic imaging", IEEE Trans. Ultrason., Ferroel., Freq. Control, vol. 54, No 8, pp.1596-1605, 2007.

[7] F.Honarvar, H.Sheikhzadeh, M. Moles and A.N.Sinclair, "Improving the time-resolution and signal-to-noise ratio of ultrasonic NDE signals", Ultrasonics, vol.41, pp.755-763, 2004.

[8] P.J.Emerson, "Alternative methods for measuring case depth in Ferrous components", British Journal of NDT, March 1976, pp.48-51.

[9] M.S.Good and J.L.Rose, "Measurement of thin case depth in hardened steel by ultrasonic pulse-echo angulation techniques", Non-destructive testing for material property determination, Hershey, pp.189-203, 1984.

[10] GB patent 1555699 , "Non-destructive testing of materials using surface ultrasonic waves generation".

[11] B.G.Martin and F.L.Becker, "The effect of near-surface metallic-property gradients on ultrasonic critical-angle reflectivity”, Materials evaluation, Jan 1980, pp.92-96.

[12] US patent 6,628,404 B1, "Acoustic sensor for real-time control for the inductive heating process".

[13] A.Abbate, J.F.Cox, S.C.Schroeder, B.Knight and J.Frankel, "Rayleigh velocities for the evaluation of coating hardness", 1996 Ultrasonics Symp., pp.1017-1020.

[14] S.E.Kruger, A.Moreau and J.-P.Monchalin, "Laser-ultrasonic monitoring of metallurgical transformations in advanced ultra-high strength steels", Proc. $1^{\text {st }}$ Int. Symp. Laser Ultrasonics: Science, Technology and Appl., Montreal, Canada, $6 \mathrm{p}$.

[15] A.Badidi Bouda, A.Benchaala, K.Alem, "Ultrasonic characterization of materials hardness", Ultrasonics, vol.38, pp.224-227, 2000.

[16] B.R.Tittmann, L.A.Ahlberg, J.M.Richardson and R.B.Thomson, "Physical property gradients from measured surface wave dispersion", IEEE Trans.Ultrason., Ferroel., Freq.Control, vol. 34, pp.500-507, 1987.

[17] A.I.Soldatov, S.A.Tsekhanovsky and R.A.Popov, "Ultrasound method", Proc. Xl Modern Technique and Technologies, pp.41-42, 2005.

[18] Methods for acoustical evaluation of metals, N.P.Alyoshin (ed.), Moscow, Machinostroenie, 1989 (in Russian).

[19] I.M.Poletika, N.M. Egorova, O.A.Kulikova, and L.B.Zuev, "Supersonic testing of mechanical property uniformity in hot-rolled steel", Technical physics, vol.46, pp.307-310, 2001.

[20] E.P.Papadakis, "Ultrasonic attenuation and velocity in three transformation products in steel", Journal of applied physics, vol.35, pp.1474-1482, 1964.

[21] B.R.Tittmann, G.A.Gordon and R.J.Tittmann, "Ultrasonic velocity and attenuation in hardened steel", Proc. Ultrasonic Int. Conf., pp.747-750, 1993.

[22] C.-H.Hsu, H.-Y.Teng, and Y.-J.Chen, “ Relationship between ultrasonic characteristics and mechanical properties of tempered matensitic stainless steel", J. Materials Eng. and Performance, vol.13, pp.593-599, 2004. 